\title{
Fast Imaging of Carbon Nanotube Carpet Growth by Environmental TEM
}

\author{
Dmitri N. Zakharov ${ }^{1}$, Mostafa Bedewy ${ }^{2}$, Viswanath Balakrishnan ${ }^{2}$, Sebastian W. Pattinson ${ }^{2}$, Eric R. \\ Meshot $^{3}$, A. John Hart ${ }^{2}$, and Eric A. Stach ${ }^{1}$ \\ 1. Center for Functional Nanomaterials, Brookhaven National Laboratory, Upton, NY 11973, USA \\ 2. The Laboratory for Manufacturing and Productivity, Massachusetts Institute of Technology, \\ Cambridge, MA 02139 USA \\ 3. Lawrence Livermore National Laboratory, Livermore, CA 94550 USA
}

Hata, et al. [1] reported a major breakthrough in carbon nanotube (CNT) growth, which allows the creation of thick ( $>1 \mathrm{um}$ ) films of single-walled CNTs to be grown from a substrate (called carpets), broadly free of other carbon allotropes. This eliminates the additional step of CNT purification often required for further studies or applications. The simplicity of the method, in conjunction with the ability to control numerous growth conditions and parameters, has made it a model setup to study fundamental mechanisms in the growth and nucleation of CNTs. It has opened a pathway for investigations concerning growth effects on CNT diameter, number of walls, and chirality dependence as a function of an array of experimental growth conditions. For example, it was shown that catalyst particles undergo Ostwald ripening and subsurface diffusion during the growth process [2]. As a result there is active catalyst mass loss that triggers accumulating growth termination of individual CNTs, followed by abrupt termination of the CNT forest growth due to the loss of a self-supporting structure [3].

Here we investigate the initial stages of CNT carpet growth, the collective behavior of nucleated CNTs, as well as the nucleation and growth of individual tubes by environmental TEM (ETEM). The ETEM imaging is done with 2.5 millisecond time resolution inside an FEI imagecorrected Titan 80-300 equipped with a Gatan K2-IS fast rate camera. The camera utilizes direct electron detection CMOS chip that has a high resistivity to electron damage and a high sensitivity capable of delivering 400 frames per second at $2 \mathrm{k} \times 2 \mathrm{k}$.

TEM samples are prepared in plan-view and cross-sectional configurations by electron beam assisted deposition of $10 \mathrm{~nm} \mathrm{Al}_{2} \mathrm{O}_{3}$ and then $1 \mathrm{~nm}$ of Fe onto $30 \mathrm{~nm}$ thick $\mathrm{SiO}_{2}$ TEM windows. It is found that as deposited, the iron is present in the form of an oxide. Metallic iron catalyst particles form upon exposure to a reducing hydrogen environment ( $40 \mathrm{mTorr}$ ) at $750^{\circ} \mathrm{C}$, however, after 30 minutes treatment only a partial reduction to metallic iron is observed (according to a series of selected area electron diffraction patterns). Further reduction is observed within the first several seconds following the introduction of acetylene $\left(10 \mathrm{mTorr}\right.$ at $\left.650^{\circ} \mathrm{C}\right)$ into the ETEM reaction chamber. Figure 1 shows frames from a movie showing the initial stages of CNT carpet growth in plan-view configuration. The cross-sectional configuration allows us to evaluate the growth rate of individual CNTs with time. It is observed that the growth rate directly correlates with changes in the CNT diameter and the generation of defects in the CNT wall (Figure 2), showing a tight correlation between CNT structure and growth modes. In general, the current ETEM setup allows us to study samples in both plan-view and cross-section configurations, to correlate CNT growth and nucleation with catalyst structure and morphology, as well as growth conditions (temperature, pressure of carbon containing precursors, reducing or buffer agents), and last but not least, correlate findings on a larger scale with ex-situ conventional CVD systems [4]. The power of this approach in understanding carpet growth will be highlighted in the presentation. 


\section{References:}

[1] K. Hata, D.N. Futaba, K. Mizuno, T. Namai, M.Yumura, S. Iijima, "Water-Assisted Highly Efficient Synthesis of Impurity-Free Single-Walled Carbon Nanotubes”, Science 306, pp. 1362 (2004)

[2] S.M. Kim, C.L. Pint, P.B. Amama, D.N. Zakharov, R.H. Hauge, B. Maruyama, and E.A. Stach, "Evolution in Catalyst Morphology Leads to Carbon Nanotube Growth Termination", J. Phys. Chem. Lett.

1, pp. 918-922 (2010)

[3] M. Bedewy, E.R. Meshot, H. Guo, E.A. Verploegen, W. Lu, A.J. Hart, “Collective Mechanism for the Evolution and Self-Termination of Vertically Aligned Carbon Nanotube Growth", J of Phys. Chem. C 113, pp. 20576-20582 (2009)

[4] Research carried out in part at the Center for Functional Nanomaterials, Brookhaven National Laboratory, which is supported by the U.S. Department of Energy, Office of Basic Energy Sciences, under Contract No. DE-SC0012704.
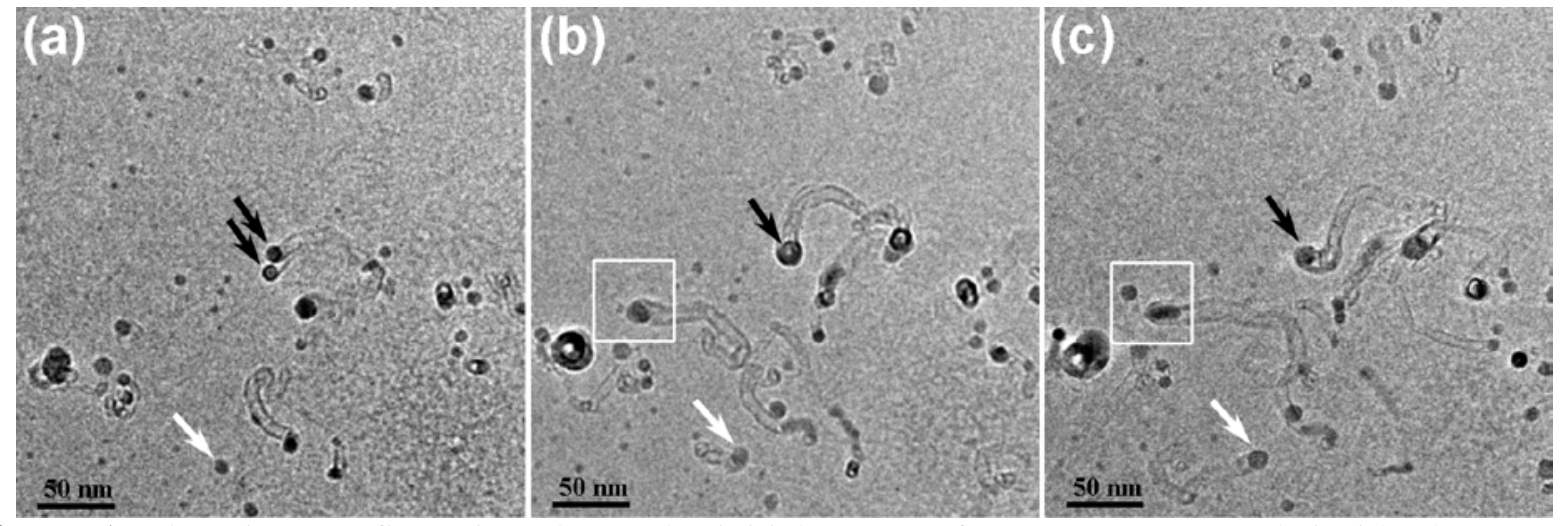

Figure 1. Plan-view configuration shows the initial stages of CNT carpet growth inside the ETEM. Individual frames (a) - (c) from a movie illustrate how both the catalyst particles and the CNTs evolve under growth conditions. Black arrows mark the merging of two catalyst particles that results in one tube terminating while the other tube continues to grow. White arrows point to a catalyst particle that has delayed tube nucleation. The white box highlight a change in the particle shape associated with the growth of a CNT and the formation of new catalyst particle.
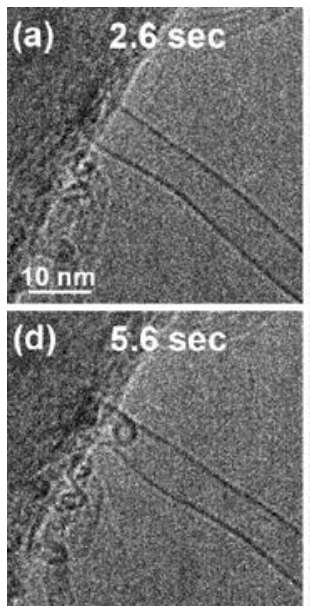
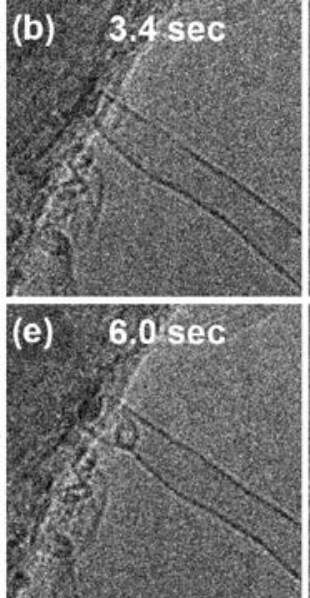
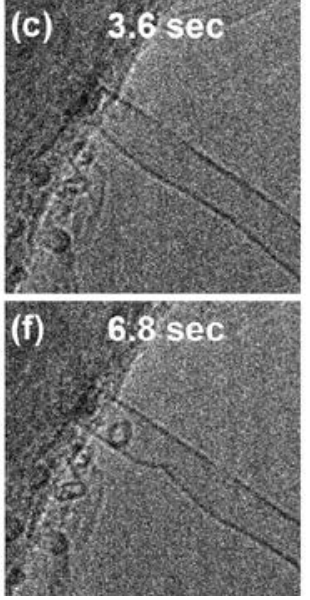

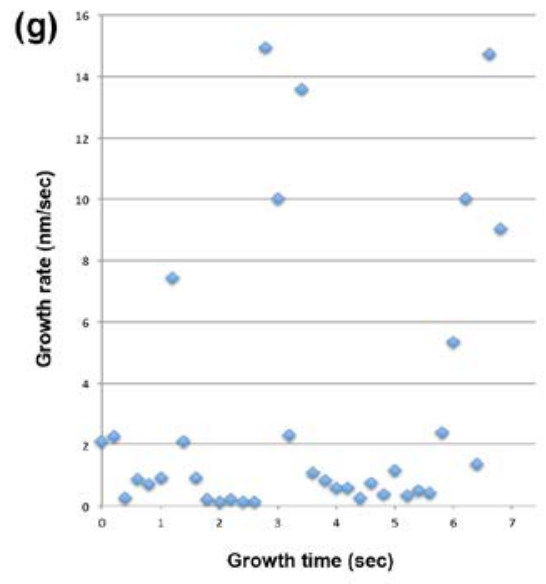

Figure 2. Individual frames (a) - (f) from a movie of individual three-walled carbon nanotube growth in cross-sectional configuration. (g) Growth rate of the nanotube versus growth time. 